\title{
Does the "Rule of Nines" Apply to Morbidly Obese Burn Victims? A Post- Bariatric Surgery Longitudinal Follow-up to the Original Patient Data Set
}

\author{
Stephen D. WOHLGEMUTH ${ }^{\mathrm{a}, \mathrm{b}}$, David STEFAN ${ }^{\mathrm{c}}$ \\ ${ }^{a}$ Eastern Virginia Medical School, Norfolk VA, USA; \\ ${ }^{\mathrm{b}}$ Sentara Comprehensive Weight Loss Solutions, Norfolk VA, USA; \\ ${ }^{\mathrm{C}}$ Novaptus Systems Inc. Chesapeake, VA, USA \\ http://dx.doi.org/10.15221/13.092
}

\section{Introduction and Background}

This paper studies longitudinal post-bariatric surgery Body Surface Area trends (BSA) for the original set of subjects that were outlined in a paper entitled "Does the "Rule of Nines" Apply to Morbidly Obese Burn Victims" published in the Journal of Burn Care Research 2013;34:447-452.

The original paper documented 150 female and 50 male preoperative morbidly obese subjects by recording height, weight, BMI. Each preoperative subject was scanned utilizing a commercially available 3D whole body scanner device.

The scanning device's measurement software was programmed to yield surface area of the torso, less the head, as well as for each arm and leg. In addition, a separate measurement algorithm determined the shape of the morbidly obese subject. In general, morbidly obese subjects can be classified as "android", whereby the largest area of excess fat is centered on the waist, "gynecoid", where the maximum distribution of fat is around the hips, or "mixed", where the distribution of excess fat is between these two extremes.

An accurate assessment of the percentage of body surface involved in a thermal injury is essential in the management of patients' with burn injuries. This has to do with the administration of fluid to promote resuscitation. Inadequate resuscitation will result in continued shock and progression of end organ damage. Excessive fluid administration puts the patient at risk of complications such as pulmonary edema. ${ }^{1}$

The "Rule of Nines" is a commonly used method of estimation of the adult Total Body Surface Area (TBSA), it was developed in 1947 by Pulaski and Tennison. ${ }^{2}$ Subjects used to derive this estimation were, back then, considered to be "average" selections within the adult population, The Rule of Nines divides the adult body into sections or multiples of $9 \%$. Each arm is considered $9 \%$ of the TBSA, each leg $18 \%$, the total trunk represents $36 \%$, the head $9 \%$, and the perineal area $1 \%{ }^{3}$. These values give guidelines as to the amount of initial fluid management, nutritional needs, classification of the degree of burn severity included a possible transfer to a specialty burn center, and provide a basis for comparative outcome data of institutions. ${ }^{4}$ There is a separate TBSA formula for infants and children. ${ }^{5}$

The adult obese patient represents a growing subset of the population. ${ }^{6}$ Morbid obesity is an extreme but not uncommon subsection of this group. A subject can be classified as morbidly obese if their BMI is 40 or over. Typical BMI values of subjects that present for weight loss surgery range from 35 , with a pressing comorbid condition, to 60 and above for both men and women. The mean BMI value for the original sample set was 45 for women, with an average weight of 265 and a height of 64 inches. The mean BMI value for men was 46, with an average weight of 319 and a height of 70 inches.

BMI may be considered an overall indicator of obesity, but it does not yield any body shape indications. A $6 \mathrm{ft}$ foot tall morbidly obese male android, with thin legs and a large abdomen may have the same BMI as a $5 \mathrm{ft}$ tall morbidly obese female gynecoid who may have most of her excess weight centered around her hips and legs.

The shape of the subject has a direct influence on the surface area of the subject's body parts. Heretofore, the shape of the subject was informally categorized by simply viewing the morbidly obese subject and opining whether he/she were "apple-ish" (android), "pear-like" (gynecoid), or somewhere in between (mixed). Figure 1 displays examples of the common shapes associated with the morbidly obese. 


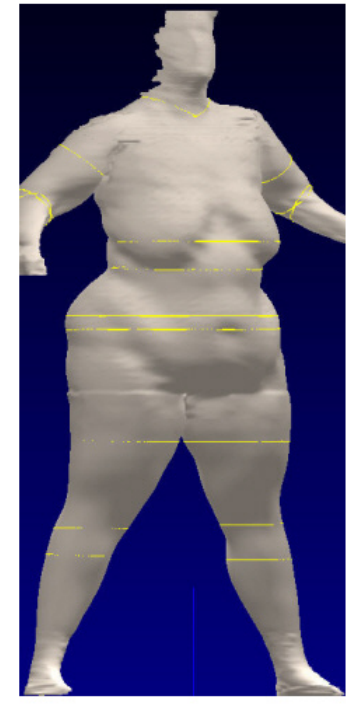

Gynecoid Shape

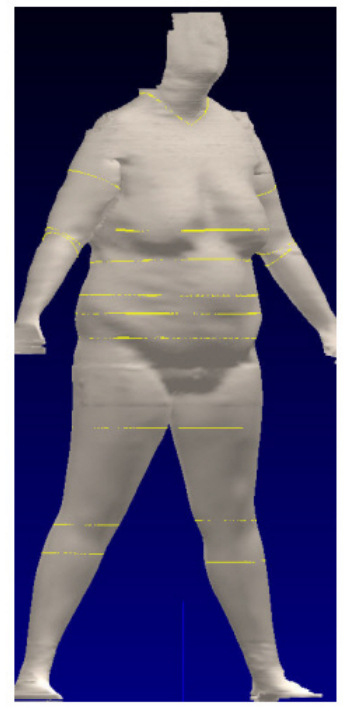

Mixed Shape

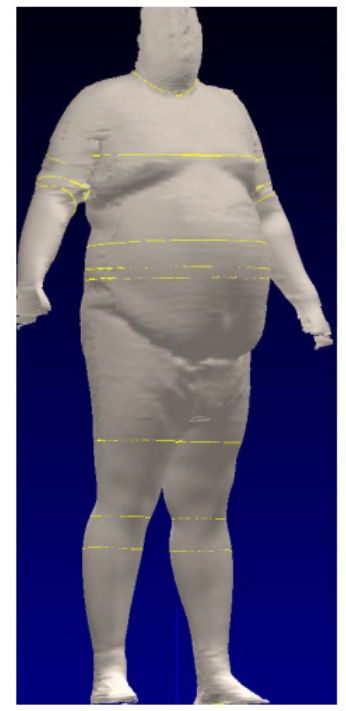

Android Shape

Figure 1. Typical Shapes for Morbidly Obese Subjects

To determine the shape of the morbidly obese subject, the torso was used as a proxy for the overall body. The torso was defined as the back of the neck to the "crotch point" of the subject, which includes the hips and buttocks. A scanner measurement profile was created that partitioned the torso into $1 \mathrm{~cm}$ segments. The circumference and the surface area for each torso segment were calculated, and each segment was then summed to calculate overall torso surface area. In addition, each segment was closed to create a segment volume. The total volume was calculated by summing up the volume of each torso segment.

The "Primary Shape" of the subject was determined by inspecting the height of the maximum torso segment circumference, from the floor of the scanner, with respect to the overall torso height. Android subjects tend to display more centralized obesity and have a maximum torso circumference closer to the waist, or center of the overall torso. Gynecoid subjects tend to have a maximum torso circumference at or near the hips.

The software was used to calculate the torso volume above this maximum torso circumference divided over the total torso volume to determine a numerical value of the Primary Shape indicator. The resultant numerical value indicated a weight distribution. In this way, the standard values of body shape indicators being equal or greater than 0.8 for gynecoid subjects, less than or equal to 0.7 for android patients, and between .71 and .79 for mixed body shapes.

Figure 2 depicts linear, circumferential, surface area and volume measurement templates applied to a female morbidly obese subject.
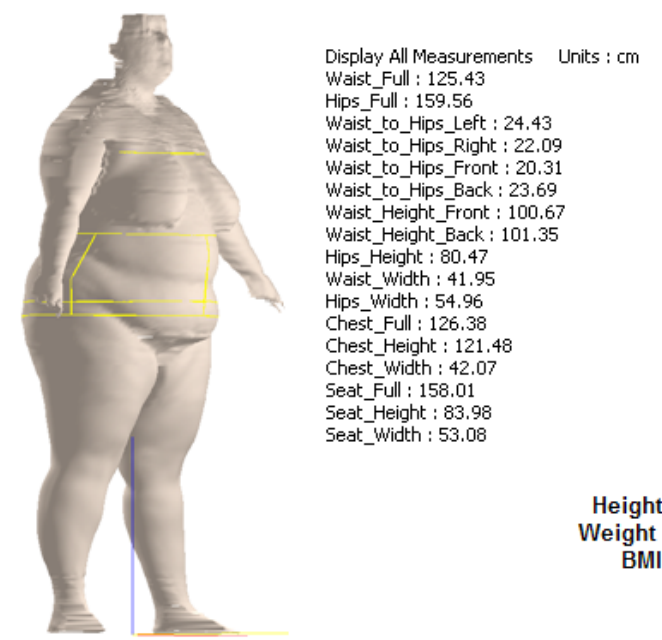

(n)

Torso Height $69 \mathrm{~cm}$



Figure 2. Extracting Measurements from Subject, including Volume and Surface Area, Maximum Torso Circumference and Primary Shape 
The commercial 3D body scanner creates a 3D body model of the subject scanned in the chamber. The body model created is essentially a collection of points in space that represent the surface of the subject with each point on the surface represented by a Cartesian coordinate, the center $(X=0, Y=0$, $Z=0$ ) being a specific spot on floor over which the subject is standing.

Once the body model is created, the scanner software searches for known landmarks on the body such as the back of the neck, the suprasternal notch, arms and leg separation. The scanner software then segments the body model into seven parts: The torso, right and left arms, right and left legs, and right and left hands.

Since the points on the surface of the body model are Cartesian in nature, the software forms triangles, or facets for the points on the surface of each body part. Summing the area of these triangles for a particular body part yields the total body part surface area.

Figure 3 depicts a body model of a scanned subject. Body parts are clearly colored to indicate separation. The measurements extracted in this case yielded bulk volume of the subject, less head, hands and feet, as well as surface area of each particular body part. It should be noted that there is a measurement for torso surface area that eliminates the surface area for the shoulders. This was used in the calculations in order to eliminate "double counting" of the surface area in which the shoulders and arms overlap.

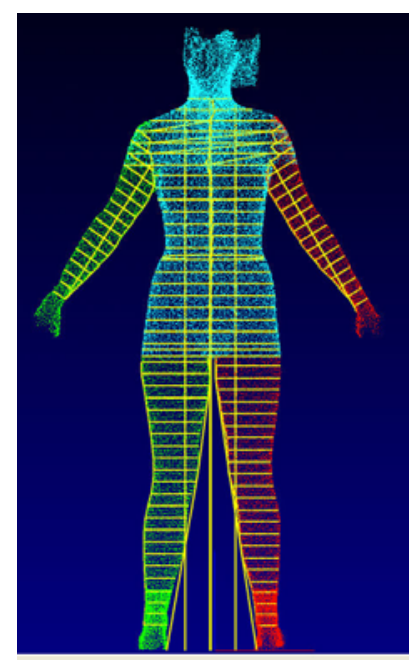

Figure 3. Body Part Separation and Measuring Bulk Volume and Surface Area Components

Thus the two components required for calculating TBSA for morbidly obese subjects, the surface area of the body parts and a mathematic determination of the subject's shape combined to yield the following summation (Table 1):

\begin{tabular}{|c|c|c|c|}
\hline & $\begin{array}{c}\text { Android } \mathrm{n}=38 \\
P=.96 \\
(95 \% \mathrm{CI},-18.760 \\
\text { to } 18.094)\end{array}$ & $\begin{array}{c}\text { Mixed } \mathrm{n}=108 \\
P=.98 \\
(95 \% \mathrm{CI},-18.242 \\
\text { to } 18.576)\end{array}$ & $\begin{array}{c}\text { Gynecoid } \mathrm{n}=54 \\
P=.98 \\
(95 \% \mathrm{CI},-17.658 \\
\text { to } 17.991)\end{array}$ \\
\hline Body mass index & $46(33-59)$ & $46(34-68)$ & $44(35-60)$ \\
\hline Weight (lbs) & 311 & 274 & 263 \\
\hline Height (inches) & 69 & 65 & 65 \\
\hline Shape indicator & 0.64 & 0.75 & 0.83 \\
\hline$\%$ Torso & $53(36)$ & $50(36)$ & $48(36)$ \\
\hline$\%$ Right arm & $7.5(9)$ & $7.5(9)$ & $7.5(9)$ \\
\hline$\%$ Left arm & $7.5(9)$ & $7.5(9)$ & $7.5(9)$ \\
\hline$\%$ Right leg & $15(18)$ & $16.5(18)$ & $17.5(18)$ \\
\hline \% Left leg & $15(18)$ & $16.5(18)$ & $17.5(18)$ \\
\hline$\%$ Head & $2(9)$ & $2(9)$ & $2(9)$ \\
\hline
\end{tabular}

Table 1. Summary of Findings for Morbidly Obese Preoperative Subjects based on Primary Shape

It should be noted that the body surface area attributed to the head was fixed at $2 \%$, building off the earlier work of Livingston and Lee, which studied 47 patients weighing more than $80 \mathrm{~kg}$. It should be also noted that Livingston and Lee's BSA values were derived using Dubois and Dubois century old equation involving linear measurements of length and circumferences of each body segment of interest. $^{\top}$ 


\section{Findings of Original Paper}

Accurately estimating the percent body surface area involved in a thermal injury is essential to the management of a burn patient. The "Rule of Nines" has traditionally been used to estimate BSA. The incidence of obesity has increased progressively, challenging the accuracy of this means of surface area estimation. This study suggests that the "Rule of Nines" is a poor approximation of TBSA, certainly for the morbidly obese patient. The findings suggest that the morbidly obese subject's shape, as measured by the primary shape indicator, to be an important index in the measurement of BSA.

The findings suggest that a "Rules of Sevens" may be a more accurate indicator of BSA for morbidly obese subjects.

\section{The Follow-on Longitudinal Study}

Subsets of the preoperative bariatric subjects used in the original paper were scanned periodically over the course of one year. These included 20 males and 20 females. Height, weight, BMI, \%EWL (excess weight loss), Primary Shape, BSA and TBSA were tabulated for 3 month, 6 month and 1 year intervals.

Questions investigated were whether these subjects, after massive weight loss, would approach the traditional "Rule of Nines" TBSA estimation, whether their overall primary shape would change or remain the same, and what post-operative time interval would yield the most dramatic change in \% BSA. The \%BSA calculations attempt to take into account as the subject weight reduces, the percentage of the BSA for the head will increase.

The calculations made the assumption that at 3 months, the \%BSA for the head would rise from $2 \%$ to $5 \%$, at six months from $5 \%$ to $7 \%$, and at 1 year the subject's $\%$ BSA for the head would approach the traditional $9 \%$ estimate.

Detailed evaluation took place on the female post-op data set, where data was available on the subjects for each 3 month, 6 month and 12 month scan.

The composite overall comparison of \% Body Surface Area over a full year post-operative period indicated that the subjects, both male and female tended towards the traditional "Rule of Nines", though at a different pace. This is mainly due to the influence of the subject's shape. Table 2 summarizes the change in \%BSA proportions for the post-operative females and males.

\begin{tabular}{|c|c|c|c|c|c|c|c|}
\hline Female & BMI & \%Head & \%Torso & \%L Arm & \% R Arm & \%L Leg & \%R Leg \\
\hline Pre-op & 45 & 2 & 51.4 & 7.2 & 7.2 & 16.1 & 16.1 \\
\hline $3 \mathrm{Mo}$ & 39 & 5 & 42.9 & 7.4 & 7.4 & 18.9 & 18.9 \\
\hline $6 \mathrm{Mo}$ & 33 & 7 & 41.1 & 7.5 & 7.5 & 18.8 & 18.8 \\
\hline $12 \mathrm{Mo}$ & 29 & 9 & 39.1 & 7.5 & 7.5 & 18.7 & 18.7 \\
\hline
\end{tabular}

\begin{tabular}{|c|c|c|c|c|c|c|c|}
\hline Male & BMI & \%Head & \%Torso & \%L Arm & \% R Arm & \%L Leg & \%R Leg \\
\hline Pre-op & 46 & 2 & 51.9 & 7.7 & 7.8 & 15.5 & 15.4 \\
\hline 3 Mo & 41 & 5 & 46.6 & 7.9 & 7.9 & 16.6 & 16.6 \\
\hline 6 Mo & 34 & 7 & 40.7 & 7.9 & 8.0 & 18.7 & 18.6 \\
\hline $12 \mathrm{Mo}$ & 28 & 9 & 38.2 & 7.9 & 8.0 & 18.6 & 18.6 \\
\hline
\end{tabular}

Table 2. Longitudinal \%BSA Results after Weight Loss Surgery

In general, the males were classified more broadly as "android" prior to surgery, with much of their excess weight distributed around the waist. These subjects' torsos represented a higher percentage of body surface area than corresponding gynecoids. At the 3 month post-operative interval, their average \%BSA derived from their torso was reduced from $52 \%$ to $46 \%$. The legs became a slightly greater percentage of TBSA.

At the $6^{\text {th }}$ month interval, the average \%BSA derived from their torso was further reduced to $41 \%$, with a corresponding increase in both leg and arm TBSA. 
At the yearly interval, the average $\%$ BSA for the torso stabilized at $38 \%$, approaching the nominal $36 \%$ BSA for the trunk of the traditional "Rule of Nines" subject. The arms approached $8 \%$ BSA while the legs maintained a value each of $19 \%$. Since the perineal area is not captured and measured by the scanner, it is possible that this region, traditionally assigned $1 \%$, could be responsible for the deviation.

The female group also tended towards the traditional "Rule of Nines", but at a much faster pace. At the 3 month post-operative interval, their average \%BSA derived from their torso was reduced from $51 \%$ to $43 \%$. The legs increased from $16 \%$ to $18 \%$ each. The decrease in $\%$ torso BSA might be attributed to the fact that the majority of the female subjects were classified as gynecoid, with their excess weight distributed mostly around their hips. As they lost weight, their hips reduced in circumference that affected the \% torso BSA more rapidly than their male android counterparts that had broader chest widths.

At the $6^{\text {th }}$ month interval, the average $\%$ BSA derived from the torso was further reduced to $41 \%$. At the yearly interval, the average \% BSA for the torso was $39 \%$, slightly more than their male counterparts. \% BSA for the arms stabilized at $7.5 \%$, and the legs at $19 \%$.

Note that the scanner doesn't accommodate surface area calculations of severely ptotic breasts or extreme skin flaps that can occur as a result of massive weight loss. In general, though, the sample set of females at the end of their yearly weight loss period did not, in general, display these conditions.

\section{Detailed Analysis of Female Data Set}

The robust information provided by the female data set has led to added insight as to the relationship between excess weight, \%excess weight loss, primary shape and \% BSA.

The average initial weight of the females was $124 \mathrm{~kg}(274 \mathrm{lbs})$. Their average height was 65 inches. Using a standard table look-up such as the Metropolitan Life Statistical Guide or other actuarial data, one can find the ideal weight of a female at 65 inches to be $62 \mathrm{~kg}$ (136 lbs). The difference between actual weight and ideal weight defines "Excess Weight", which is a standard monitoring component. A subject is said to have "manifested" the benefits of weight loss surgery if they achieve $75 \%$ excess weight loss within 1 year's time.

The average excess weight of the pre-operative female sample set was $49 \%$, fairly indicative of the overall female subjects that present for weight loss surgery. This corresponds to having to lose on average, $62 \mathrm{~kg}$ to achieve ideal weight. The average BMI of the group was 46 . The composite primary shape of the pre-operative female subjects was 0.77 . This overall primary shape value suggests that the aggregate shape of the subjects were mixed, but tending toward gynecoid (defined as 0.80 or above).

At the 3rd month interval, the average weight loss for the group was $20 \mathrm{~kg}$ (45 lbs). The average BMI was 39, and the \% Excess Weight for the group was 39\%. The group's primary shape indicator changed from .77 to .86 indicating that as the women lost weight they began to regain common female proportions. The $\%$ BSA of the group's torso declined from $51 \%$ to $43 \%$.

Within the 3 to $6^{\text {th }}$ month interval, the average weight loss for the group was $16 \mathrm{~kg}$. This rate declined from the $3^{\text {rd }}$ month interval of $20 \mathrm{~kg}$. Average BMl decreased to 33, and the Excess Weight for the group declined to $29 \%$. A BMI of less than 35 indicates that, as a group, the females are below the lower end of the range considered to be morbidly obese.

The group's primary shape indicator remained stable at 0.86 . This indicates that at a certain point, even though weight loss has occurred, the weight loss is in proportion to the body shape. The \% BSA of the group's torso declined to $41 \%$, a slower rate of decline than the $3^{\text {rd }}$ month interval.

Finally, at the yearly interval, the average weight loss for the group from the $6^{\text {th }}$ month interval was 11 $\mathrm{kg}(25 \mathrm{lbs})$, a declining rate from the previous interval. The overall weight loss is $46 \mathrm{~kg}$. This corresponds to an overall excess weight loss percentage of $74.2 \%$, very close to the $75 \%$ weight loss marker.

The average BMI for the group was 29 , which is considered "overweight" but not obese. The group's primary shape indicator remained at 0.86 and the $\%$ BSA of the group's torso was $39 \%$. 


\section{Discussion and Conclusions}

Morbidly obese subjects have body shapes that do not correspond well to the "Rule of Nines"; a "Rule of Sevens" seems to be more appropriate. However, morbidly obese subjects that undergo massive weight loss after surgery do, after time, approach the parameters for estimating \%BSA as given by the traditional guidelines.

The Excess Weight, or its derivative, \% Excess Weight Loss appears to be an excellent indicator as to when the morbidly obese subject's \% BSA begins to tend toward normalization. At some point in the weight loss cycle, the Excess Weight reduces to such a point that \%BSA begins to revert towards traditional surface area proportions. BMI has a weight component implicit in its value, and may be used as a proxy for Excess Weight.

Since severely burned victims are often in a trauma center, quick decisions must be made based on known entities such as height and weight. From the analysis of the data, it appears that if the subject's BMI is over 40, the "Rule of Sevens" should be the guideline. If the subject's BMI is under 40 , the "Rule of Nines" appears to be more applicable.

\section{References}

1. Livingston $\mathrm{EH}$, Lee $\mathrm{S}$. Percentage if burned body surface area determination in obese and nonobese patients. J. Surg Res 2000;91:106-10.

2. Knaysi GA, Crikelair GF, Cosman B. The rule of nines: its history and accuracy. Plast Reconstr Surg 1968;41:560-3.

3. Sabiston DC. Textbook of surgery: The biological basis of modern surgical practice, $13^{\text {th }}$ ed., Philadelphia: Saunders; 1986

4. Monafo WW. Initial management of burns. N. Engl J Med 1999;21:1581.

5. Lund C, Browder N. The estimation of area of burns. Surg Gynecol Obstret 1944:79:352.

6. Ogden CL, Carroll MD, Curtin LR, McDowell MA, Tabak CJ, Felgal KM. Prevalence of overweight and obesity in the United States, 1999-2004. JAMA 2006;295(13):1549-55.

7. DuBois $D$, DuBois $E$. A formula to estimate the approximate surface area if height and weight be known. Arch Intern Med 1915;17:863-71. 